\title{
High-Dose Compound Heat Map for 3D-Cultured Glioblastoma Multiforme Cells in a Micropillar and Microwell Chip Platform
}

\author{
Dong Woo Lee, ${ }^{1}$ Sang-Yun Lee, ${ }^{2}$ Il Doh, ${ }^{3}$ Gyu Ha Ryu, ${ }^{4}$ and Do-Hyun Nam ${ }^{2,5}$ \\ ${ }^{1}$ Department of Biomedical Engineering, Konyang University, Daejeon 35365, Republic of Korea \\ ${ }^{2}$ Department of Health Sciences and Technology, SAIHST, Sungkyunkwan University, Seoul 06351, Republic of Korea \\ ${ }^{3}$ Center for Medical Metrology, Korea Research Institute of Standards and Science (KRISS), Daejeon, Republic of Korea \\ ${ }^{4}$ Office of R\&D Strategy \& Planning, Samsung Medical Center, Seoul, Republic of Korea \\ ${ }^{5}$ Department of Neurosurgery, Samsung Medical Center, Sungkyunkwan University, School of Medicine, Seoul 06351, Republic of Korea
}

Correspondence should be addressed to Gyu Ha Ryu; gyuha.ryu@samsung.com and Do-Hyun Nam; nsnam@skku.edu

Received 24 February 2017; Revised 9 June 2017; Accepted 20 June 2017; Published 18 October 2017

Academic Editor: Sara Piccirillo

Copyright (c) 2017 Dong Woo Lee et al. This is an open access article distributed under the Creative Commons Attribution License, which permits unrestricted use, distribution, and reproduction in any medium, provided the original work is properly cited.

Glioblastoma multiforme (GBM) is recognized as the most common and lethal form of central nervous system cancer. To cure GBM patients, many target-specific chemotherapeutic agents have been developing. However, 2D monolayer cell-based toxicity and efficacy tests did not efficiently screen agents due to the pool reflection of in vivo microenvironments (cell-to-cell and cellto-extracellular matrix interaction). In this study, we used a 3D cell-based, high-throughput screening method reflecting the microenvironments using a micropillar and microwell chip platform to draw a high-dose heat map of the cytotoxicity and efficacy of 70 compounds, with two DMSO controls. Moreover, the high-dose heat map model compared the responses of four 3D-cultured patient-derived GBM cells and astrocytes to high dosages of compounds with respect to efficacy and cytotoxicity, respectively, to discern the most efficacious drug for GBM. Among the 70 compounds tested, cediranib (a potent inhibitor of vascular endothelial growth factor (VEGF) receptor tyrosine kinases) exhibited the lowest cytotoxicity to astrocytes and high efficacy to GBM cells in a high-dose heat map model.

\section{Introduction}

Glioblastoma multiforme (GBM) is the most common, aggressive, and lethal primary malignant brain tumor that stems from astrocytes. These tumors are usually highly malignant because the cells can metastasize from the primary tumor without detection and invade the surrounding normal brain tissue to form new tumor "satellites" that lead to tumor recurrence [1]. The current standard of care is surgical resection coupled with ionizing radiation (IR) and the chemotherapeutic agent temozolomide $\left(\right.$ Temodar $^{\circledR}$, Temodal $^{\circledR}$, TMZ) $[2$, $3]$. However, this treatment only provides patients with GBM a 12-14-month survival period after diagnosis [2, 3]. Despite aggressive surgical resection and chemotherapy, almost all patients with GBM present with tumor recurrence. Thus, many target-specific or general-chemotherapeutic agents have been developed to cure patients with GBM. Although some of the compounds exhibit good efficacy toward GBM, the resulting cytotoxicity of normal glial cells in the central nervous system has been an issue. To measure cytotoxicity of compounds in normal glial cells, neural stem cells or astrocytes are used $[4,5]$. Astrocytes are the most abundant member of the glial family and have a wide range of adaptive functions in the central nervous system (CNS). They interact with neurons, provide structural, metabolic, and trophic support, participate in synaptic activity, mediate ionic and transmitter homeostasis, and regulate blood flow [6, 7]. Since astrocytes play an important role in the CNS, treatmentinduced toxicity of the CNS remains a major cause of morbidity in patients with cancer [8]. Thus, a high-dose heat map model comparing the responses of high-dose compounds on astrocytes and GBM cells is required to validate the most efficacious drugs toward GBM. Previous high-dose heat map models using 2D cell-based high-throughput screening are well developed $[9,10]$. However, because $2 \mathrm{D}$ cell-based assay 
does not fully reflect in vivo microenvironments (cell-to-cell and cell-to-extracellular matrix interaction), a 3D cell-based assay was used to screen compounds [11-15], including our previously developed system [13-17]. Especially, 3D cultured astrocyte and GBMs show more in vivo like model [1820]. Thus, assay based on $3 \mathrm{D}$ cultured astrocyte and GBMs with high-throughput manner may give new potential to screen GBM target agents. Our previous system [13-17] shows successfully data of 3D cell-based assays with highthroughput manner by comparing their own data with $2 \mathrm{D}$ cell-based assay [13], gene [14], and clinical data [17]. By applying the abovementioned quantitative 3D-cultured cellassay platform, astrocytes and patient-derived GBM cells were $3 \mathrm{D}$-cultured and screened to select the most represented compounds that were not cytotoxic to normal brain cells and were particularly efficient for patient-derived GBM cells. Figure 1 shows 3D cell-based high-throughput screening chips culturing three-dimensionally four GBM cells and astrocyte. Since TMZ is a representative drug used in the treatment of patients with GBM, it was used as a control compound to verify the high-dose heat map. By comparing TMZ with 69 other compounds, compounds in the high-dose heat map were tested for cytotoxicity and efficacy in GBM cells.

\section{Materials and Methods}

2.1. Astrocyte and Patient-Derived Cell Culture. We purchased NHA-astrocyte AGM (LONZA, Cat. number cc2565). Astrocyte was cultured with ABM Basal media (LONZA, Cat. number cc-3187) added with AGM SingleQuot Kit Suppl.\&Growth Factors (LONZA, Cat. number cc4123). Patient-derived GBM cells were obtained from GBM patients who underwent brain tumor removal surgery at the Samsung Medical Center (Seoul, Korea). Informed consent was obtained from all patients. Following a previously reported procedure [13], surgical samples were enzymatically dissociated into single cells. Four patient-derived cells were obtained from four GBM patients. Dissociated GBM cells were cultured in cell culture flasks (from Eppendorf, T-75) filled with Neurobasal A (NBA) conditioned media. The NBA conditioned media comprised N2 and B27 supplements $(0.53$ each; Invitrogen) and human recombinant bFGF and EGF (25 ng/ml each; R\&D Systems), hereafter, referred to as NBE condition media. Cell flasks were placed in a humidified 5\% $\mathrm{CO}_{2}$ incubator (Sheldon Mfg., Inc.) at $37^{\circ} \mathrm{C}$. The cells were routinely passed every 4 days at $70 \%$ confluence. For the experiment, the cell suspensions were collected in a $50 \mathrm{ml}$ falcon tube from the culture flask. GBM cells were then suspended in $5 \mathrm{~mL}$ of NBE condition media. After centrifugation at $2000 \mathrm{rpm}$ for $3 \mathrm{~min}$, the supernatant was removed, and the cells were resuspended with NBA conditioned media to a final concentration of $10 \times 10^{6}$ cells $/ \mathrm{mL}$. The number of cells in the NBA conditioned media was calculated with the AccuChip automatic cell counting kit (Digital Bio, Inc.). The rest of the cells were seeded at a concentration of $2 \times 10^{6}$ cells in a T-75 flask containing $15 \mathrm{~mL}$ of NBA conditioned media.
2.2. Chip Layout and Experimental Procedure. The basic layout of the micropillar and microwell chip for a 12-compound screening is shown in Figure 2. The microwell chip is divided into 72 lines, and each line has 6 microwells for replicates. For compound analysis, approximately 100 cells (patient-derived GBM cells) in $50 \mathrm{~nL}$ with a $0.75 \%$ alginate concentration by volume $(0.75 \mathrm{w} / \mathrm{w})$ were automatically dispensed onto a micropillar chip by using ASFA $^{\mathrm{TM}}$ Spotter ST (Medical \& Bio Device, South Korea). The ASFA Spotter ST uses a solenoid valve (The Lee Company, USA) for dispensing the $50 \mathrm{~nL}$ droplets of the cell-alginate mixture and $1 \mu \mathrm{L}$ of media or compounds. After dispensing the cells, as shown in Figure 1(b), the micropillar chip containing human cells in alginate was sandwiched (or "stamped") with the microwell chip for 3D cell culture and compound efficacy tests. A single chip can screen 72 compounds with 6 replicates simultaneously. A micropillar chip with alginate dispensed on each pillar spot and the microwell chip containing 72 compounds are shown in Figure 2. The micropillar chip with cells dispensed is stamped together with its complementary microwell chip comprising 532 wells that are $1.2 \mathrm{~mm}$ in diameter. One microliter of growth media was dispensed into each microwell. The micropillar and microwell chip in the combined form are shown in Figure 1(b). After 1 day of incubation at $37^{\circ} \mathrm{C}$ to stabilize the cells, the micropillar chip containing the cells was moved to a new microwell chip filled with various test compounds. Next, the combined chips were incubated for 3 days, as shown in Figure 1(b). Cell viability against the compounds was measured with Calcein AM live cell staining dye ( $4 \mathrm{mM}$ stock from Invitrogen), which stains viable cells with green fluorescence. The staining dye solution was prepared by adding $1.0 \mu \mathrm{L}$ of Calcein AM ( $4 \mathrm{mM}$ stock from Invitrogen) to $8 \mathrm{~mL}$ staining buffer (MBDSTA50, Medical \& Bio Device, South Korea). To measure cell viability quantitatively after staining the alginate spots, cells on the micropillar chip were scanned. As shown in Figure 1(a), scanned images were obtained with an automatic optical fluorescence scanner (ASFA Scanner ST, Medical \& Bio Device, South Korea).

2.3. Workflow of High-Dose Compound Heat Map. The highdose compound heat map model for measuring cytotoxicity and efficacy in GBM cells was quantified using a 3D cellbased screening using a micropillar and microwell chip. The workflow of high-dose compound heat map is shown in Figure 3. 72 compounds with high dosage, including two DMSO controls, were screened against astrocytes and GBM cells for measuring toxicity and efficacy, respectively. Based on astrocyte cytotoxicity and efficacy of GBM cells, compounds were divided into four groups. For this heat map, we selected the most promising compounds that exhibited less cytotoxicity toward astrocytes and high efficacy toward GBM cells.

\section{Results and Discussion}

To determine the cytotoxicity against normal glial cells, we treated astrocytes (represented as normal glial cells) to high 


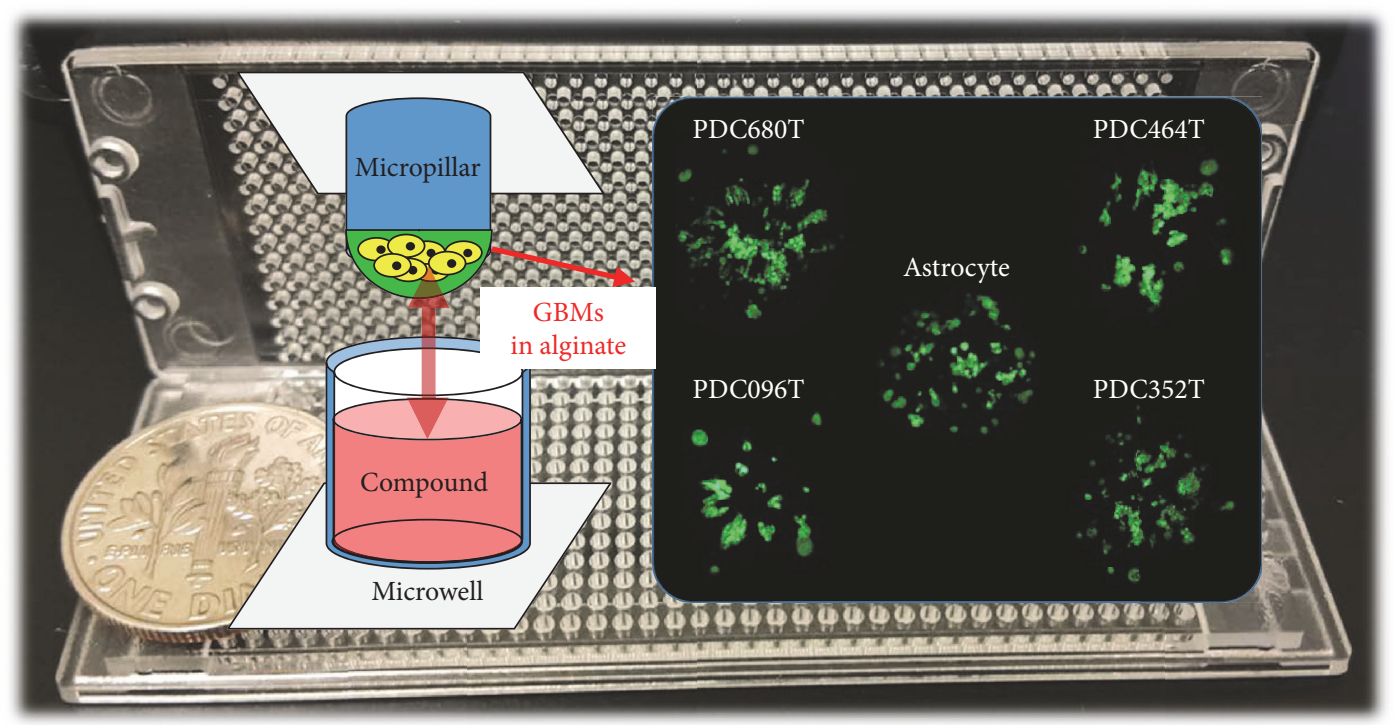

(a)

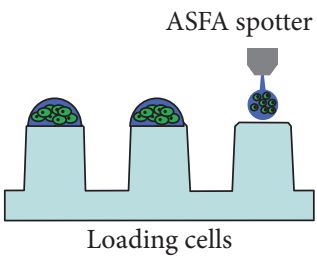

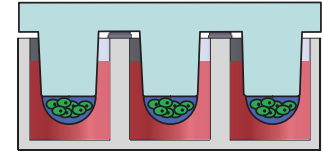

1 day before incubation

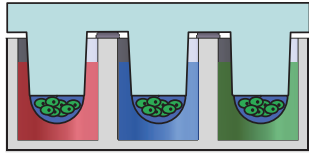

Compound exposure

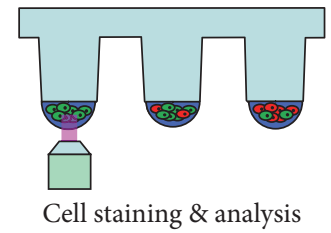

(b)

FIGURE 1: 3D cell-based high-throughput screening chips. (a) Photo and schematic view of micropillar and microwell chip platform. Green dots are 3D-cultured astrocytes and glioblastoma multiforme (GBM) cells in alginate spot on the micropillar. (b) Schematic view of the experimental procedure. Cells are dispensed and immobilized in alginate onto the top of the micropillars and dipped in the microwells containing growth media for 1-day culture by sandwiching the micropillar and microwell chips. Compounds are dispensed into the microwells and cells are exposed to the compounds by moving the micropillar chip to a new microwell chip. 3D-cultured cells are stained with Calcein $\mathrm{AM}$, and the dried alginate spot on the micropillar chip is scanned for data analysis.

dosages $(20 \mu \mathrm{M})$ of 72 compounds. Among the 72 compounds tested, TMZ is one of most popular drugs for GBM. The lipophilic nature of TMZ permits it to penetrate the blood-brain barrier and, thereby, allows it to be administered orally. It has also been approved by the US FDA for use in the treatment of refractory anaplastic astrocytoma in adults since 1999 and in newly diagnosed adult patients with GBM since 2005. Thus, we used TMZ as a control compound for comparing cytotoxicity of compounds toward astrocytes. We evaluated cytotoxicity of other compounds in comparison to TMZ and evaluated the efficacy of these compounds with patient-derived GBM cells.

3.1. Toxicity of High-Dose Compounds in Astrocytes. The viability of astrocytes after exposure to 72 compounds (including 2 DMSO control) after the 3- and 7-day treatment is shown in Figure 4. 16 compounds, including TMZ, exhibited high astrocyte viability and increased to $>50 \%$ after the 3 -day compound treatment. On the seventh day, only 7 compounds exhibited $>50 \%$ astrocyte viability: cediranib, INCB28060, nilotinib, LDE225, sotrastaurin, vismodegib, and amoral exhibited low toxicity (with astrocyte viability $>50 \%$ ). However, TMZ, which is widely known for its low cytotoxicity, exhibited high toxicity (with astrocyte viability $<18 \%$ ) after the 7-day compound treatment. $20 \mathrm{uM}$ of TMZ is high dose and TMZ shows high toxicity for long-day culture (7 days). Thus, based on the 3-day compound treatment, we evaluated the cytotoxicity of the compounds by comparing the viability of astrocyte after exposure with other compounds and with TMZ (over 90\%). Among 16 compounds, 7 exhibited similar astrocyte viability to TMZ, cediranib, INCB28060, ABT-888, dabrafenib, vismodegib, and amoral and therefore may be good candidates to test the efficacy against patient-derived GBM cells.

\subsection{Efficacy Test of 70 Compounds for Patient-Derived GBM} Cells. The viabilities of astrocyte and four patient-derived GBM cells after the 3-day compound treatment are shown in Figure 5. Most of the targeted compounds exhibited high efficacy with $20 \mathrm{uM}$ dosages. By comparing efficacy of GBM cells 

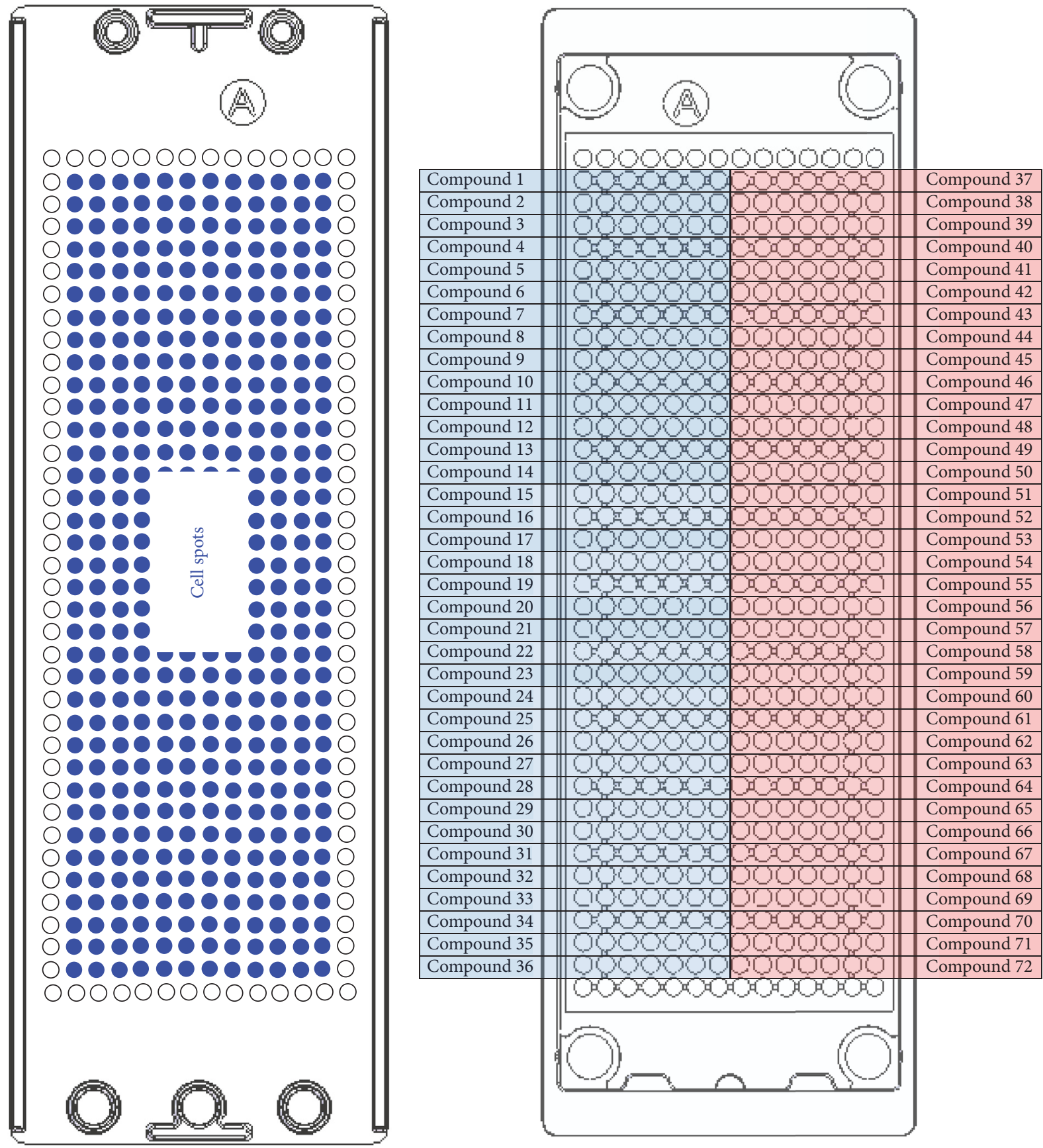

FIGURE 2: Chip layout for high-dose 72 (including 2 DMSO controls)-compound heat map. Each compound has seven replicates.

and cytotoxicity of astrocytes, most of the compounds exhibited nonspecific, high toxicity for both GBM cells and astrocytes. Among seven nontoxic compounds, INCB28060, ABT888 , vismodegib, and amoral did not suppress the patientderived GBM cells. Thus, they may be ineffective compounds in treating GBM. TMZ, cediranib, and dabrafenib exhibited low GBM cell viability in more than one patient-derived GBM cell. In particular, cediranib exhibited high efficacy in all four patient-derived GBM cells and exhibited no cytotoxicity toward astrocytes, while TMZ showed high efficacy in only
\#1 GBM cell among the four patient-derived GBM cells. Previous studies [21, 22] have shown that cediranib is a potent oral inhibitor of vascular endothelial growth factor (VEGF) receptors and demonstrates improved progressionfree survival in an uncontrolled phase II study of patients with recurrent glioblastoma. The drug is administered orally and once daily and has a manageable side-effect profile. In addition, it has potent antiedema and steroid-sparing effects that may improve the quality of life of patients with GBM. Several clinical trials are ongoing testing cediranib 


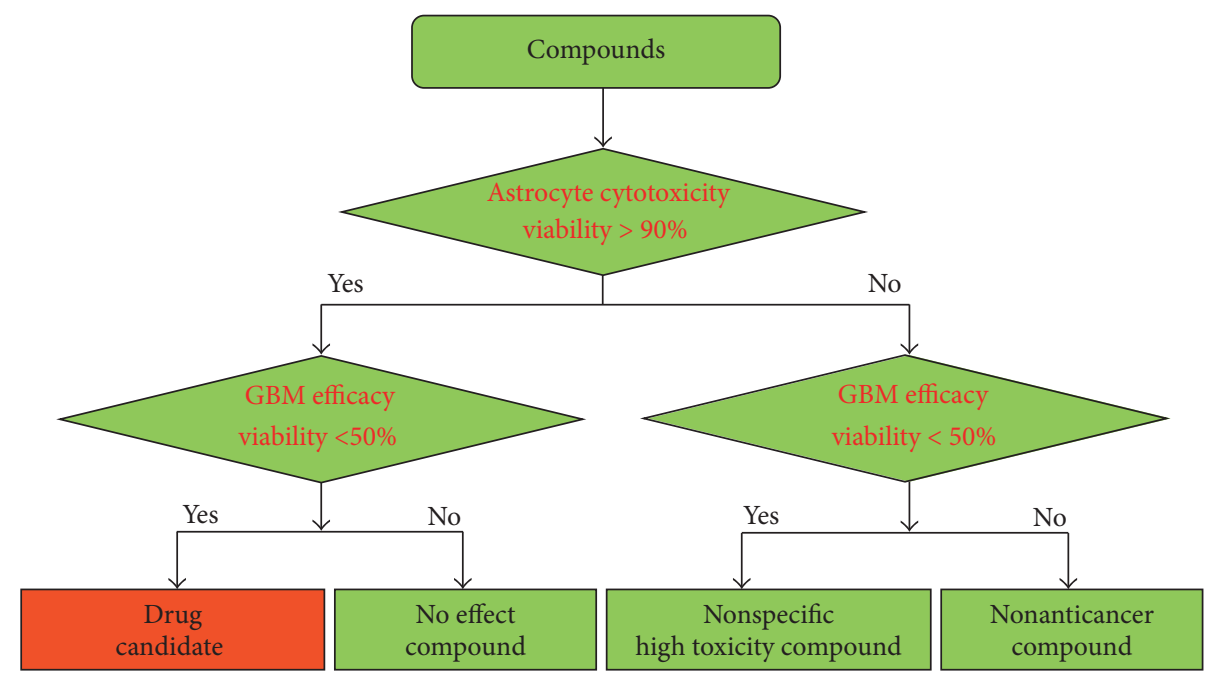

\section{\begin{tabular}{|l||l|l||l|}
\hline Promote to next step & No-go decision & Promote to next step & No-go decision
\end{tabular}}

FIGURE 3: High-dose compound heat map for selecting low cytotoxicity and high efficacy compound.

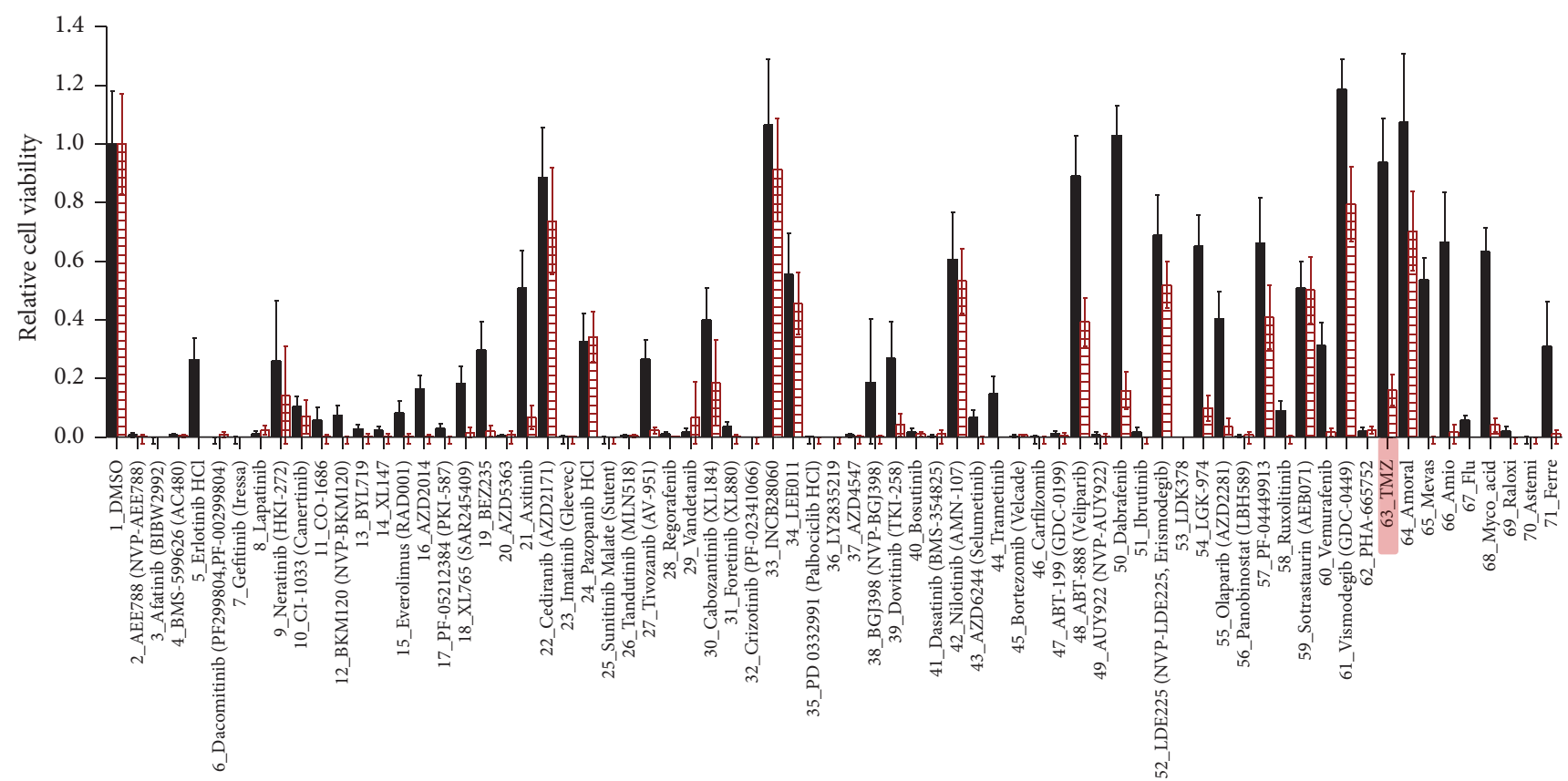

Astrocyte_3 days

Astrocyte_7 days

FIGURE 4: Relative cell viability of astrocytes after exposure to 70 compounds after the 3-and 7-day compound treatment. Cell viabilities are calculated from 3D cell size.

in patients with gliomas. Through this $3 \mathrm{D}$ cell-based highdose heat map model, we could also identify cediranib as a valid compound for proof-of-concept of heat map model. So, this $3 \mathrm{D}$ cell-based high-dose heat map could narrow down drug candidates for GMBs. As further study, we need to draw dose response curve to measure $\mathrm{IC}_{50}$ in $\mathrm{GBMs}$ about agents showing low toxicity and high efficacy.

\section{Conclusion}

We used a 3D cell-based, high-throughput screening technique using a micropillar and microwell chip platform to determine the effects of high dosage compounds on the cytotoxicity and efficacy on astrocytes and GBM cells, which were graphically represented in a high-dose heat map model. 


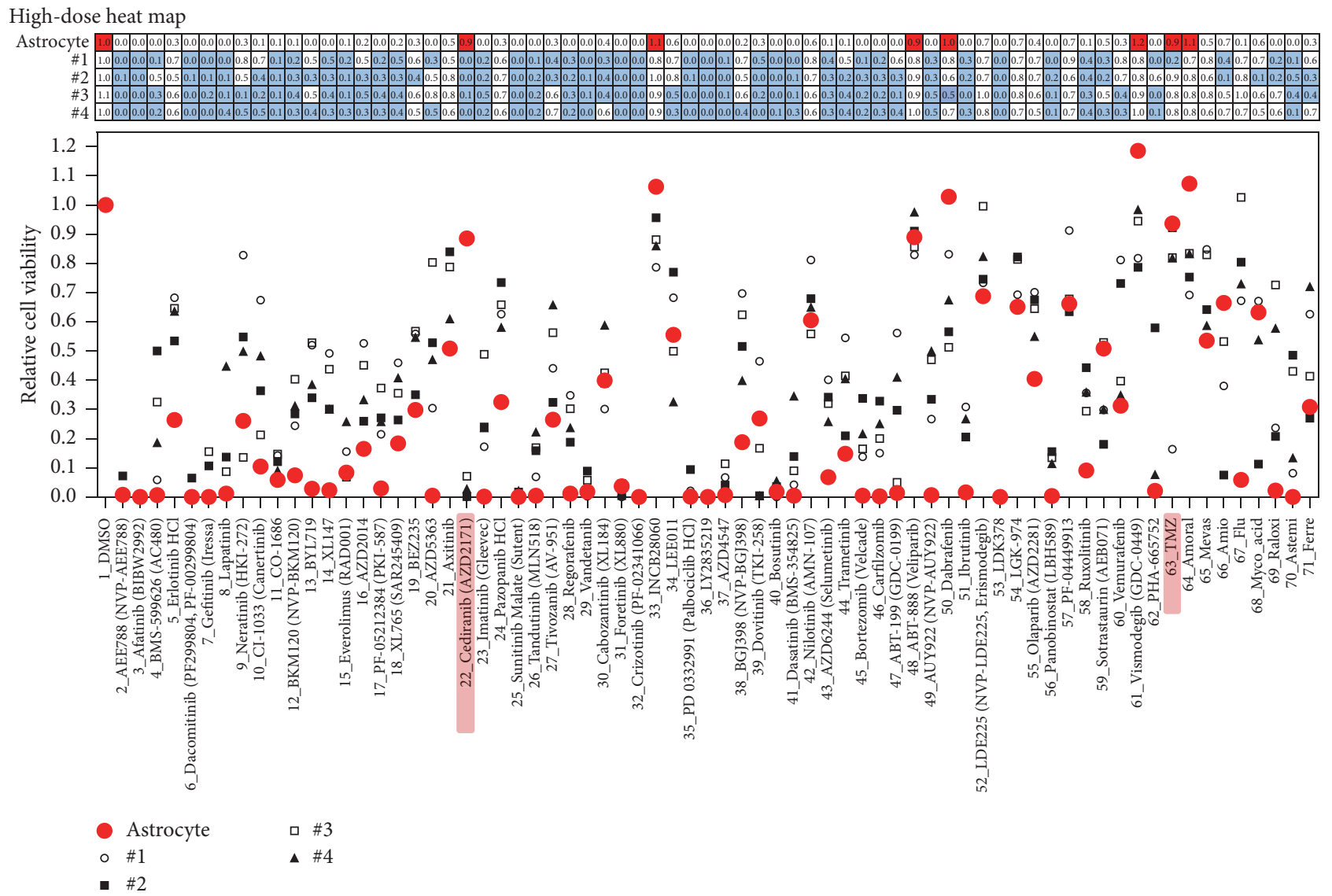

FIGURE 5: High-dose compound heat map. Astrocytes and four patient-derived glioblastoma multiforme (GBM) cells were treated with 70 compounds for 3 days. In the high-dose heat map (table over graph), red solid filling denotes astrocytes with viability $>90 \%$ and blue solid filling denotes GBM cells with viability $<50 \%$.

Although some compounds exhibited good efficacy toward GBM, cytotoxicity toward normal glial cells in the central nervous system was an impending issue. Thus, we used a high-dose heat map model and considered both cytotoxicity and efficacy by comparing the response of highdose compound on 3D-cultured GBM cells and astrocytes to screen the most efficacious drugs for GBM. Seventy compounds with dosage of $20 \mathrm{uM}$, including specific-target general-chemotherapeutic agents, were used against four patient-derived GBM cells and astrocytes to compare the cytotoxicity and efficacy of the compounds. For simulating an in vivo microenvironment, GBM cells and astrocytes were encapsulated with alginate and 3D-cultured in a micropillar and microwell chip platform. Among the 70 compounds tested, cediranib exhibited the lowest cytotoxicity toward astrocyte and showed high efficacy toward GBM. Thus, in early stage of the drug development, the micropillar and microwell chip platform could culture patient-derived GBMs with 3D manner and be used for screening toxicity and efficacy of many lead compounds targeted GBMs before animal tests or clinical trials.

\section{Conflicts of Interest}

The authors declared no potential conflicts of interest with respect to the research, authorship, and/or publication of this article.

\section{Acknowledgments}

This research was supported by the Bio \& Medical Technology Development Program of the NRF funded by the Korean Government, MSIP (NRF-2015M3A9A7029740).

\section{References}

[1] C. L. Smith, O. Kilic, P. Schiapparelli et al., "Migration phenotype of brain-cancer cells predicts patient outcomes," Cell Reports, vol. 15, no. 12, pp. 2616-2624, 2016.

[2] U. Linz, "Commentary on effects of radiotherapy with concomitant and adjuvant temozolomide versus radiotherapy alone on survival in glioblastoma in a randomised phase III study: 5-Year analysis of the EORTC-NCIC trial (Lancet Oncol. 2009;10:459466)," Cancer, vol. 116, no. 8, pp. 1844-1846, 2010.

[3] R. Stupp, W. P. Mason, and M. J. van den Bent, "Radiotherapy plus concomitant and adjuvant temozolomide for glioblastoma," Oncology Times, vol. 27, no. 9, pp. 15-16, 2005.

[4] G. Yount, L. Soroceanu, H.-J. Wang et al., "Selective toxicity of a highly potent camptothecin analogue: a pilot study with glioblastoma multiforme cells," Anticancer Research, vol. 36, no. 11, pp. 5845-5848, 2016.

[5] S. Dolma, H. J. Selvadurai, X. Lan et al., "Inhibition of dopamine receptor D4 impedes autophagic flux, proliferation, and survival of glioblastoma stem cells," Cancer Cell, vol. 29, no. 6, pp. 859-873, 2016. 
[6] I. Markiewicz and B. Lukomska, "The role of astrocytes in the physiology and pathology of the central nervous system," Acta Neurobiol Exp (Wars), vol. 66, no. 4, pp. 343-358, 2006.

[7] E. O'Brien, C. Howarth, and N. R. Sibson, "The role of astrocytes in CNS tumours: pre-clinical models and novel imaging approaches," Frontiers in Cellular Neuroscience, vol. 7, no. 40, pp. $1-13,2013$.

[8] C. Soussain, D. Ricard, J. R. Fike, J.-J. Mazeron, D. Psimaras, and J.-Y. Delattre, "CNS complications of radiotherapy and chemotherapy," The Lancet, vol. 374, no. 9701, pp. 1639-1651, 2009.

[9] R. H. Shoemaker, "The NCI60 human tumour cell line anticancer drug screen," Nature Reviews Cancer, vol. 6, no. 10, pp. 813-823, 2006.

[10] J.-P. Mpindi, P. Swapnil, B. Dmitrii et al., "Impact of normalization methods on high-throughput screening data with high hit rates and drug testing with dose-response data," Bioinformatics, vol. 31, no. 23, pp. 3815-3821, 2015.

[11] M.-Y. Lee, R. A. Kumar, S. M. Sukumaran, M. G. Hogg, D. S. Clark, and J. S. Dordick, "Three-dimensional cellular microarray for high-throughput toxicology assays," Proceedings of the National Academy of Sciences of the United States of America, vol. 105, no. 1, pp. 59-63, 2008.

[12] Y.-C. Tung, A. Y. Hsiao, S. G. Allen, Y.-S. Torisawa, M. Ho, and S. Takayama, "High-throughput 3D spheroid culture and drug testing using a 384 hanging drop array," Analyst, vol. 136, no. 3 , pp. 473-478, 2011.

[13] D. W. Lee, S. H. Yi, S. H. Jeong, B. Ku, J. Kim, and M.-Y. Lee, "Plastic pillar inserts for three-dimensional (3D) cell cultures in 96-well plates," Sensors and Actuators, B: Chemical, vol. 177, no. 1, pp. 78-85, 2013.

[14] D. W. Lee, Y.-S. Choi, Y. J. Seo et al., "High-throughput screening (HTS) of anticancer drug efficacy on a micropillar/microwell chip platform," Analytical Chemistry, vol. 86, no. 1, pp. 535-542, 2014.

[15] D. W. Lee, Y.-S. Choi, Y. J. Seo et al., "High-throughput, miniaturized clonogenic analysis of a limiting dilution assay on a micropillar/microwell chip with brain tumor cells," Small, vol. 10, no. 24, pp. 5098-5105, 2014.

[16] D. W. Lee, M.-Y. Lee, B. Ku, and D.-H. Nam, "Automatic 3D cell analysis in high-throughput microarray using micropillar and microwell chips," Journal of Biomolecular Screening, vol. 20, no. 9, pp. 1178-1184, 2015.

[17] D. W. Lee, S. Lee, L. Park et al., "High-throughput clonogenic analysis of 3D-Cultured patient-derived cells with a micropillar and microwell chip," Slas Discovery: Advancing Life Sciences $R \nLeftarrow D$, vol. 22, no. 5, pp. 645-651, 2017.

[18] A. M. Pasca, S. A. Sloan, L. E. Clarke et al., "Functional cortical neurons and astrocytes from human pluripotent stem cells in 3D culture," Nature Methods, vol. 12, no. 7, pp. 671-678, 2015.

[19] N. Gomez-Roman, K. Stevenson, L. Gilmour, G. Hamilton, and A. J. Chalmers, "A novel 3D human glioblastoma cell culture system for modeling drug and radiation responses," NeuroOncology, vol. 19, no. 2, pp. 229-241, 2017.

[20] E. East, N. Johns, M. Georgiou et al., "A 3D in vitro model reveals differences in the astrocyte response elicited by potential stem cell therapies for CNS injury," Regenerative Medicine, vol. 8, no. 6, pp. 739-746, 2013.

[21] J. Dietrich, D. Wang, and T. T. Batchelor, "Cediranib: profile of a novel anti-angiogenic agent in patients with glioblastoma," Expert Opinion on Investigational Drugs, vol. 18, no. 10, pp. 15491557, 2009.
[22] T. T. Batchelor, E. R. Gerstner, K. E. Emblem et al., "Improved tumor oxygenation and survival in glioblastoma patients who show increased blood perfusion after cediranib and chemoradiation," Proceedings of the National Academy of Sciences, vol. 110, no. 47, pp. 19059-19064, 2013. 


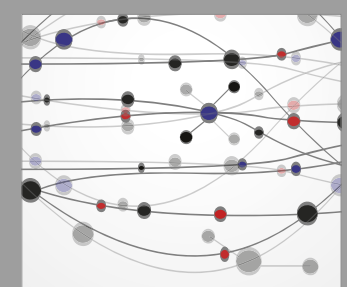

The Scientific World Journal
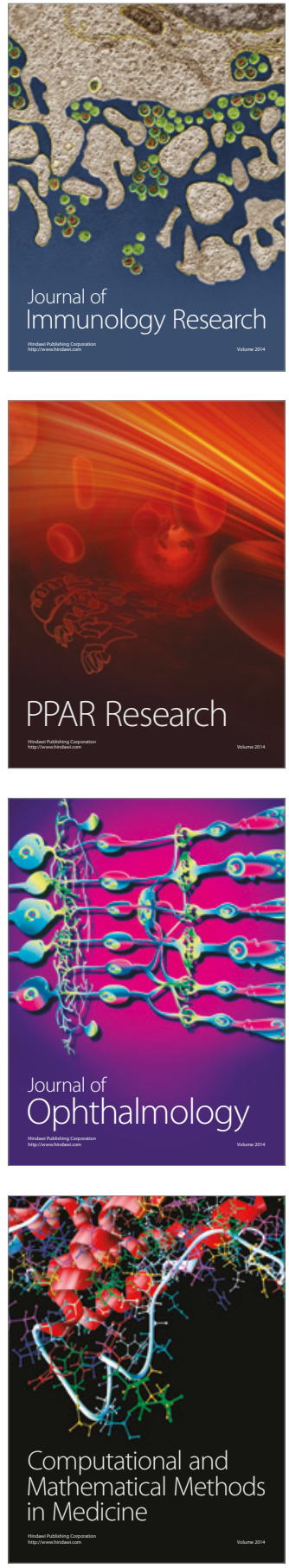

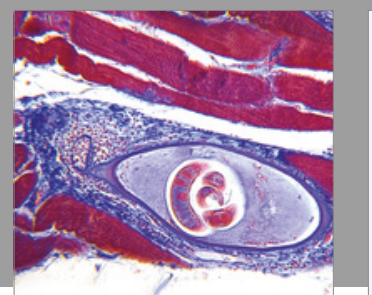

Gastroenterology Research and Practice
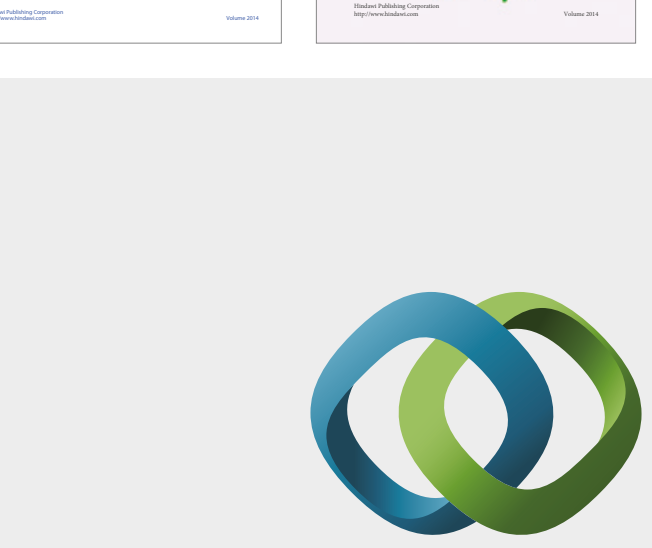

\section{Hindawi}

Submit your manuscripts at

https://www.hindawi.com
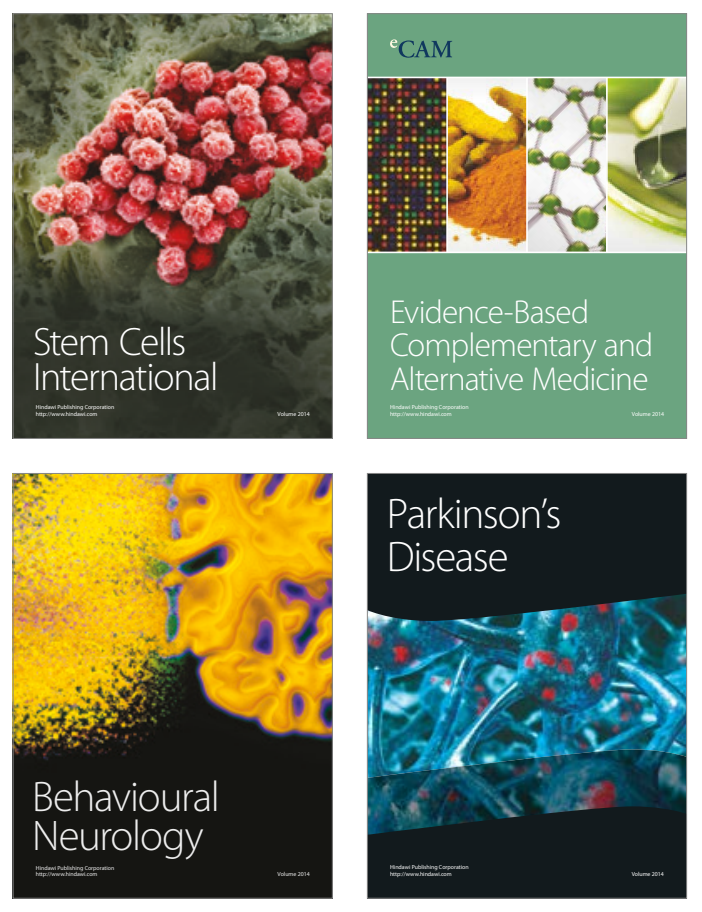
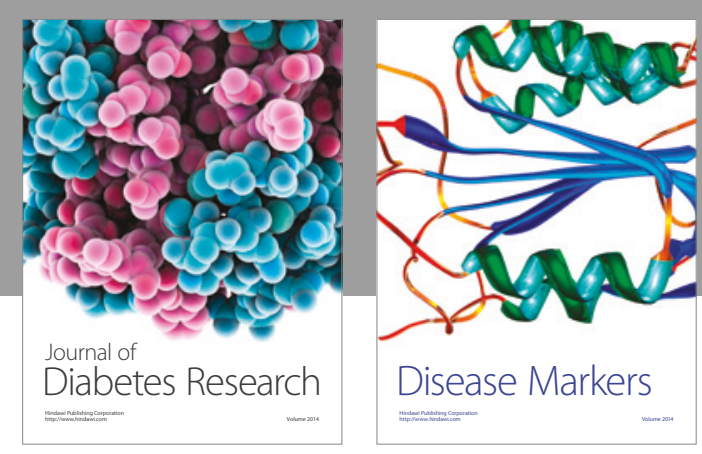

Disease Markers
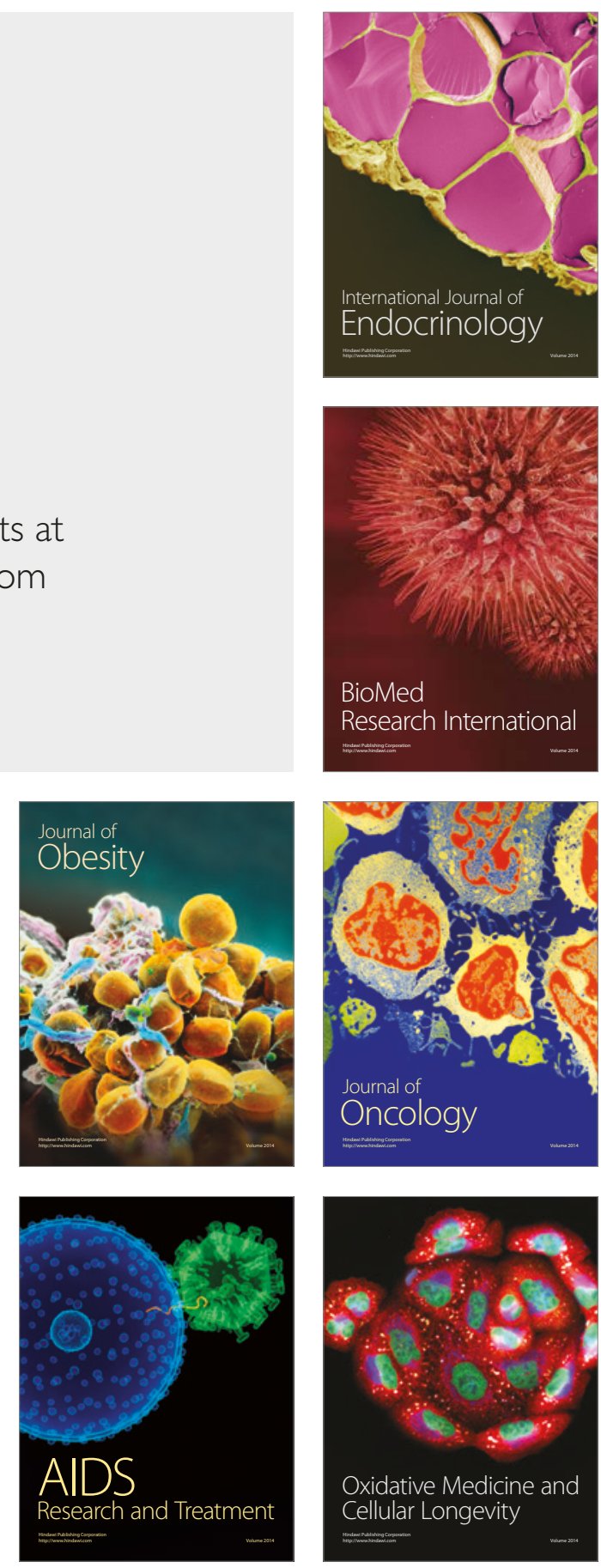